relationship between age and PDI scores in non-clinical populations. The increased tendency toward delusional ideation during adolescence needs to be clarified. Multidimensional approaches to psychosis proneness based on the developmental characteristics of adolescents are needed for the detection of high-risk group.

References

[1] Peters, E.R., Joseph, S.A. \& Garety, P.A. (1999a) Measurement of delusional ideation in the normal population: introducing the PDI (Peters et al. Delusions Inventory). Schizophr Bull 25, 553-76.

[2] Verdoux, H., van Os, J., Maurice-Tison, S., Gay, B., Salamon, R. \& Bourgeois, M. (1998b) Is early adulthood a critical development stage for psychosis proneness? A survey of delusional ideation in normal subjects. Schizophr Res 29, 247-254.

\section{8 - MASS MEDIA EFFECT ON CASE RECRUITMENT FOR STUDY ON PSYCHOPATHOLOGICAL PROGRESS OF EARLY SCHIZOPHRENIA-LIKE DISORDER (SOPRES) IN TAIWAN}

Chen-Chung Liu, Yen-Nan Chiu, Meng-Chuan Lai, Hai-Gwo Hwu Department of Psychiatry, National Taiwan University Hospital, Taipei, Taiwan

chchliu@ntu.edu.tw

Introduction: To recruit subjects suspected as early schizophrenia-like disorder (ESLD), a special clinic for "cognitive perceptual disturbance of adolescent and young adult" was launched by the study hospital in Nov. 2006. A large flow of attendees came after several major newspapers reported a prodromal case's favorable outcome released by our research team in mid Aug. 2007.

Methods: The differences in demographic characteristics, tentative clinical impressions, proportion of eligible subjects and actual participation in our study before and after the report were compared by Chi square and t-test statistics.

Results: Before the media report, 84 new attendees visited our special clinic in 33 sessions with 54 of them eligible and 49 actually recruited. Soon after the report, 49 new attendees in 8 sessions, on average 2 years younger, with 16 eligible and 13 recruited. The clinical impressions are more diverse immediately after the report. The number of new attendees fell to the baseline later on (2.9 per visit).

Conclusions: Information reported by mass media did draw people's attention and prompt them to come for assessment. The effect only lasted for two months, though, and the number of eligible subjects did not increase proportionately. The diversity of clinical impressions among the news-drawn attendees suggests an unmet need for psychoeducation and psychiatric services. For a relatively uncommon and ill-defined clinical entity, the prodromal state, we might need an innovative approach other than this type of propagation to facilitate targeted audience's self-identification and referral.

Acknowledgements: Supported by NHRI, Taiwan

References

[1] Hafner, H., K. Maurer, et al. (2004). "Early detection and secondary prevention of psychosis: facts and visions." Eur Arch Psychiatry Clin Neurosci 254(2): 117-28.

[2] Heinssen, R.K., B.N. Cuthbert, et al. (2003). "Overcoming barriers to research ine arly serious mental illness: issues for future collaboration." Schizophr Bull 29(4): 737-45.

[3] Johannessen, J.O., T.H. McGlashan, et al. (2001). "Early detection strategies for untreated first-episode psychosis." Schizophr Res 51(1): 39-46.

[4] McGlas han, T.H., J. Addington, et al. (2007). "Recruitment and treatment practices for help-seeking "prodromal" patients." Schizophr Bull 33(3): 715-26.

[5] McGorry, P.D., A.R. Yung, et al. (2003). "The "close-in" or ultra high-risk model: a safe and effecti ve strategy for research and clinical intervention in prepsychotic mental disorder." Schizophr Bull 29(4): 771-90.

[6] Peindl, K.S. and K.L. Wisner (2003). "Successful recruitment strategies for women in postpartum mental health trials." J Psychiatr Res 37(2): 117-25.

\section{9 - PRODROMAL SYMPTOMS IN GENETICALLY AT RISK YOUTH}

R.P. Rajarethinam $^{1}$, Michelle Buda Abela ${ }^{1}$, Diana Dworakowski ${ }^{2}$, Debra Montrose $^{2}$, Jean Meiwald ${ }^{2}$, Matcheri Keshavan ${ }^{3}$

${ }^{1}$ Wayne State University, Detroit; ${ }^{2}$ University of Pittsburgh,

Pittsburgh; ${ }^{3}$ Wayne State University and University of Pittsburgh,

Detroit, USA

rrajaret@med.wayne.edu

Introduction: Clinical and research interest in the prodromal stage of schizophrenia offers hope. As a genetically vulnerable youngsters during the developmental age of risk pose a unique research opportunity. Children and adolescents at genetic risk for schizophrenia offer a valuable population to investigate the premorbid precursors of this illness. In this study, we examined the presence prevalence of prodromal symptoms in a cohort of young genetically at risk individuals and studied the relationship of these symptoms with other premorbid behavioral and neurocognitive deficiencies deficits prevalent in at risk population.

Methods: 39 adolescent non-psychotic relatives of patients with schizophrenia participated in this study at the University of Pittsburgh (mean age $15.8+3.6,54 \%$ female and 62\% African American). Following informed consent, they went through a comprehensive diagnostic, neuropsychiatric, and cognitive evaluation. including SCID, Kiddie-SA DS PL, Soft Signs, olfactory identification test, Social Anhedonia Scale, Scale for Prodromal Symptoms, Premorbid Adjustment Scale, IQ and computerized neuropsychological tests to assess verbal memory, spatial working memory, Wisconsin Card Sort Test and Continuous Performance Task (IP). Cognitive processes evaluated measures included attention, verbal memory, working memory, processing speed, and executive functioning. Prodromal symptoms were assessed by the Structured Interview for Prodromal Symptoms and Scale of Prodromal Symptoms.

Results: 13 individuals $(33 \%)$ exhibited prodromal symptoms with a score of more than 2. Verbal memory deficits, poor premorbid functioning and poor global assessment of functioning correlated strongly with the presence of premorbid symptoms. Deficits in verbal memory, attention, processing speed, and abstract reasoning were significantly correlated with the presence of prodromal symptoms. Prodromal symptoms were also correlated with neurological impairments, problems with premorbid social functioning, and poorer overall global functioning.

Conclusions: Our data suggest that there may be a subgroupa substantial proportion of genetically at risk individuals who exhibit subtle prodromal symptoms who be appear more prone to develop future psychosis. In addition, this subgroup may exhibit be characterized by subtle neurocognitive deficits and decline in functioning long before development of prodromal symptoms or psychosis. The predictive validity of these markers for later emergence of schizophrenia can only be ascertained by follow up studies.

Acknowledgements: This work was supported in part by NIMH grants MH 64023, 01180 (MSK), a NARSAD independent Investigator aw ard (MSK), NARSAD and GCRC grant M01 RR00056.

\section{0 - SEXUAL FUNCTIONING IN THE AT RISK MENTAL STATE AND FIRST EPISODE SCHIZOPHRENIA}

$\underline{\text { Tiago Reis Marques }}^{1}$, R.M. Murray ${ }^{1}$, P.K. McGuire ${ }^{1}$, S. Shubulade ${ }^{2}$, O.D. Howes ${ }^{1}$

${ }^{1}$ Institute of Psychiatry, London; ${ }^{2}$ Institute of Psychiatry/OASIS,

London, UK

tiago.marques@iop.kcl.ac.uk

Introduction: Sexual functioning is a major concern in schizophrenia and studies has shown high rates of sexual dysfunction. However, is not clear whether sexual dysfunction is secondary to illness or other factors such as antipsychotic drugs. In this study we measured sexual function and psychopathology in two unmedicated samples: one with prodromal symptoms and other with first episode schizophrenia. 